\title{
ON THE ORBIT TYPES IN A CIRCLE ACTION
}

\author{
ELLIOTT STEIN ${ }^{1}$
}

ABSTRACT. An example is constructed of a smooth $S^{1}$ action on $S^{5}$ (or $R^{5}$ )
which has more orbit types than its slice representation at a fixed point.

In [3, p. 353], Frank Raymond posed the following question: If $S^{1}$ acts on $\mathbf{R}^{n}$ fixing $x$, and $H$ is an isotropy subgroup of the action, is $x$ in the closure of the set of orbits of type $H$ ? In the context of smooth actions, this amounts to asking whether the slice representation at $x$ precisely determines the orbit types in the action. An affirmative answer, in general, would constitute a very strong regularity theorem for $S^{1}$ actions. However, we will show here that the answer can be negative. Specifically, we construct a smooth $S^{1}$ action on $S^{5}$ having a circle as fixed point set, with normal slice representation $t^{m} \oplus t^{n}$ ( $t=$ the standard generator of the complex representation ring of $S^{1}$ ), but having an orbit of type $\mathbf{Z}_{m n}$. By deleting a single fixed point, we obtain the example relevant to Raymond's question, with $H=\mathbf{Z}_{m n}$. (We are assuming that $m$ and $n$ are greater than one and relatively prime.)

The idea of the construction is simply to start with a likely candidate for the orbit space, and then build the action one piece at a time. The orbit space we use is a four-sphere which contains an immersed two-sphere, $i\left(S^{2}\right)$, in general position and with one self-intersection point. If this intersection is thought of as coming from the fact that $i$ (north pole) $=i$ (south pole), then this point will represent the orbit of type $\mathbf{Z}_{m n}$. The fixed points are $i$ (equator), and the remaining two components of $i\left(S^{2}\right)$ represent orbits of types $\mathbf{Z}_{m}$ and $\mathbf{Z}_{n}$. The principal orbits are the points in the complement of $i\left(S^{2}\right)$.

Let $p: D^{4} \rightarrow D^{3}$ be the cone over the Hopf map. Take a proper, unknotted $\operatorname{arc}(I, \partial I) \subset\left(D^{3}, S^{2}\right)$ such that $I$ does not contain $0 \in D^{3}$, and let $N$ be a closed tubular neighborhood of $I$ which also misses 0 . Set $U=p^{-1}(N)$, and $X=\overline{D^{4} \sim U}$. (In terms of the description in the previous paragraph, $X$ is the complement of an open tubular neighborhood of $i\left(S^{2}\right)$.) Clearly, $X$ is a smooth manifold with boundary, and $\partial X$ is a principal $S^{1}$ bundle over $\partial\left(\overline{D^{3} \sim N}\right)=S^{1} \times S^{1}$. Set $\partial_{+} X=X \cap U \subset \partial X$. Looking at the MayerVietoris sequence for the union $D^{4}=X \cup U$, the following facts can be easily verified: (a) $H^{i}(X)=\mathbf{Z}$ for $i=0,1,2$; (b) $H^{i}(X)=0$ for $i \geqslant 3$; (c)

Received by the editors October 28, 1976 and, in revised form, December 17, 1976.

AMS (MOS) subject classifications (1970). Primary 57E15, 57E25.

${ }^{1}$ Partially supported by the National Science Foundation. 
the restriction $H^{2}(X) \rightarrow H^{2}\left(\partial_{+} X\right)$ is injective. From this last fact, we conclude that $H^{2}(X) \rightarrow H^{2}(\partial X)$ is injective. From the exact sequence of the pair $(X, \partial X)$, it follows that $H^{2}(\partial X)=\mathbf{Z} \oplus \mathbf{Z}$.

We can now explicitly describe the manifold $\partial X$ in a way that will be useful in the construction. Any principal $S^{1}$ bundle over $S^{1} \times S^{1}$ is classified by an Euler class. For each $t \in H^{2}\left(S^{1} \times S^{1}\right)=Z$, we have a total space, $E(t)$, satisfying $H^{2}(E(t))=\mathbf{Z} \oplus \mathbf{Z} \oplus \mathbf{Z}_{t}$. The manifolds $E(t)$ and $E(-t)$ are diffeomorphic. We can write

$$
E(t)=S^{1} \times S^{1} \times I /(x, y, 0) \sim\left(x y^{t}, y, 1\right) .
$$

Thus, $\partial X \simeq E(1) \simeq E(-1)$; henceforth, we will think of $\partial X$ as

$$
S^{1} \times S^{1} \times I /(x, y, 0) \sim\left(x y^{-1}, y, 1\right) .
$$

We also identify $\partial_{+} X$ with $S^{1} \times S^{1} \times\left[\frac{1}{3}, \frac{2}{3}\right]$.

Now define the following $S^{1}$-spaces ( $S^{1}$ and $D^{2}$ are considered as the usual subsets of the complex numbers), with $m, n, p$, and $q$ integers satisfying $p m+q n=1$ and $m, n>1$ :

$A=X \times S^{1}$ with the action $g \circ(x, z)=(x, g z)$;

$B_{1}=S^{1} \times S^{1} \times\left[\frac{1}{3}, \frac{4}{9}\right] \times D^{2}$ with the action $g \circ(x, y, t, z)=\left(x, g^{m} y, t\right.$, $\left.g^{n} z\right)$

$B_{2}=S^{1} \times S^{1} \times\left[\frac{5}{9}, \frac{2}{3}\right] \times D^{2}$ with the action $g \circ(x, y, t, z)=\left(x, g^{n} y, t\right.$, $g^{\left.-m_{z}\right) \text {. }}$

We have $\partial A=\partial X \times S^{1}$. Let

$\partial_{+} A=\partial_{+} X \times S^{1}, \partial_{+} B_{1}=S^{1} \times S^{1} \times\left[\frac{1}{3}, \frac{4}{9}\right] \times S^{1}, \quad$ and $\partial_{+} B_{2}=S^{1} \times S^{1} \times\left[\frac{5}{9}, \frac{2}{3}\right] \times S^{1}$.

Form the $S^{1}$-space $M$ by attaching $B_{1}$ and $B_{2}$ to $A$ along $\partial_{+} A$ by the following equivariant diffeomorphisms:

$$
\begin{aligned}
f_{1}: \partial_{+} B_{1}= & S^{1} \times S^{1} \times\left[\frac{1}{3}, \frac{4}{9}\right] \times S^{1} \rightarrow S^{1} \times S^{1} \times\left[\frac{1}{3}, \frac{4}{9}\right] \\
\times & S^{1} \subset \partial_{+} A \\
& (x, y, t, z) \rightarrow\left(x, y^{-n_{z} m}, t, y^{p_{z} q}\right) \\
f_{2}: \partial_{+} B_{2}= & S^{1} \times S^{1} \times\left[\frac{5}{9}, \frac{2}{3}\right] \times S^{1} \rightarrow S^{1} \times S^{1} \times\left[\frac{5}{9}, \frac{2}{3}\right] \\
& \times S^{1} \subset \partial_{+} A \\
& (x, y, t, z) \rightarrow\left(x, y^{m} z^{n}, t, y^{q_{z}-p}\right) .
\end{aligned}
$$

Using van Kampen's theorem and the Mayer-Vietoris sequence, it can be verified that $M$ is 2-connected.

Now we need to describe $\partial M$ as an $S^{1}$-space. One piece, denoted $\partial_{1} M$, is 


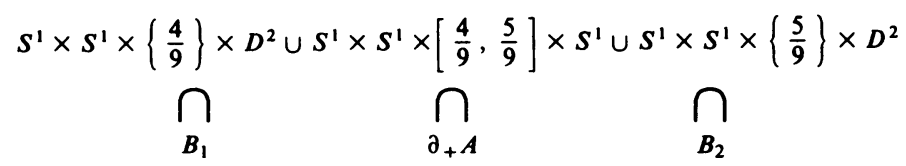

If we regard $S^{1} \times S^{1} \times\left[\frac{4}{9}, \frac{5}{9}\right] \times S^{1}$ as a collar of the boundary of $S^{1} \times S^{1} \times\left\{\frac{4}{9}\right\} \times D^{2}$, then we can write (abusing the notation slightly)

$$
\partial_{1} M=S^{1} \times S^{1} \times\left\{\frac{4}{9}\right\} \times D^{2} \underset{f_{2}^{-1} \circ f_{1}}{\bigcup} S^{1} \times S^{1} \times\left\{\frac{5}{9}\right\} \times D^{2} .
$$

Since $f_{2}^{-1} \circ f_{1}: S^{1} \times S^{1} \times S^{1} \rightarrow S^{1} \times S^{1} \times S^{1}$ is given by $(x, y, z) \rightarrow(x, z$, $\left.y^{-1}\right)$, it is easy to see that $\partial_{1} M$ is just $S^{1} \times S^{3}$ with the $S^{1}$ action given by $g \circ\left(z, w_{1}, w_{2}\right)=\left(z, g^{m} w_{1}, g^{n} w_{2}\right)$, where $|z|=\left|w_{1}\right|^{2}+\left|w_{2}\right|^{2}=1$.

The other piece of $\partial M$, denoted $\partial_{2} M$ is

$$
S^{1} \times S^{1} \times\left\{\frac{1}{3}\right\} \times D^{2} \cup\left(\partial A \sim \partial_{+} A\right) \cup S^{1} \times S^{1} \times\left\{\frac{2}{3}\right\} \times D^{2}
$$

From the description of $\partial X$ given above, it is clear that

$$
\partial A=S^{1} \times S^{1} \times I \times S^{1} /(x, y, 0, z) \sim\left(x y^{-1}, y, 1, z\right) .
$$

Let $\alpha: S^{1} \times S^{1} \times S^{1} \rightarrow S^{1} \times S^{1} \times S^{1}$ be given by $\alpha(x, y, z)=\left(x y^{-1}, y, z\right)$. We can now express $\partial_{2} M$ as

$$
S^{1} \times S^{1} \times\left\{\frac{1}{3}\right\} \times D^{2} \underset{f_{2}^{-1} \circ \alpha \circ f_{1}}{\bigcup} S^{1} \times S^{1} \times\left\{\frac{2}{3}\right\} \times D^{2} .
$$

A short computation shows that $f_{2}^{-1} \circ \alpha \circ f_{1}: S^{1} \times S^{1} \times S^{1} \rightarrow S^{1} \times S^{1} \times$ $S^{1}$ is given by $(x, y, z) \rightarrow\left(x y^{n} z^{-m}, z, y^{-1}\right)$.

Now let $C_{1}=S^{1} \times S^{1} \times D^{2}$ with the $S^{1}$ action $g \circ(x, y, z)=\left(g^{m n} x\right.$, $\left.g^{m} y, g^{n} z\right)$. Let $C_{2}=S^{1} \times S^{1} \times D^{2}$ with the action, $g \circ(x, y, z)=\left(g^{m n} x\right.$, $\left.g^{n} y, g^{-m} z\right)$. There is an equivariant diffeomorphism $\lambda: \partial C_{1} \rightarrow \partial C_{2}$, given by $\lambda(x, y, z)=\left(x, z, y^{-1}\right)$. If we form the space $C_{1} \cup_{\lambda} C_{2}$, we get $S^{1} \times S^{3}$ with the action given by $g^{\circ}\left(z, w_{1}, w_{2}\right)=\left(g^{m n} z, g^{m} w_{1}, g^{n} w_{2}\right)$. However, there are equivariant diffeomorphisms, $\theta_{1}: S^{1} \times S^{1} \times\left\{\frac{1}{3}\right\} \times D^{2} \rightarrow C_{1}$ and $\theta_{2}$ : $S^{1} \times S^{1} \times\left\{\frac{2}{3}\right\} \times D^{2} \rightarrow C_{2}$, given by $\theta_{1}\left(x, y, \frac{1}{3}, z\right)=\left(x y^{n}, y, z\right)$ and $\theta_{2}(x, y$, $\left.\frac{2}{3}, z\right)=\left(x y^{m}, y, z\right)$. We conclude that $C_{1} \cup_{\lambda} C_{2}$ is equivariantly diffeomorphic to

$$
S^{1} \times S^{1} \times\left\{\frac{1}{3}\right\} \times D^{2} \underset{\theta_{2}^{-1} \circ \lambda \circ \theta_{1}}{\bigcup} S^{1} \times S^{1} \times\left\{\frac{2}{3}\right\} \times D^{2} .
$$

Another short computation shows that $\theta_{2}^{-1} \circ \lambda \circ \theta_{1}=f_{2}^{-1} \circ \alpha \circ f_{1}$. Therefore, $\partial_{2} M=S^{1} \times S^{3}$ with the action $g \circ\left(z, w_{1}, w_{2}\right)=\left(g^{m n} z, g^{m} w_{1}, g^{n} w_{2}\right)$.

Let $D_{1}=S^{1} \times D^{4}$ with the action $g \circ\left(z, w_{1}, w_{2}\right)=\left(z, g^{m} w_{1}, g^{n} w_{2}\right)$, where $\left|w_{1}\right|^{2}+\left|w_{2}\right|^{2} \leqslant 1$. Let $D_{2}=S^{1} \times D^{4}$ with the action $g \circ\left(z, w_{1}, w_{2}\right)=\left(g^{m n} z\right.$, $\left.g^{m} w_{1}, g^{n} w_{2}\right)$. There are obvious equivariant diffeomorphisms $\partial D_{1} \rightarrow \partial_{1} M$ and $\partial D_{2} \rightarrow \partial_{2} M$. Form the closed $S^{1}$-manifold $\Sigma^{5}=M \cup D_{1} \cup D_{2}$. Again, using van Kampen's theorem and the Mayer-Vietoris sequence, it can be verified 
that $\pi_{1} \Sigma^{5}=H_{2}\left(\Sigma^{5}\right)=0$. It follows immediately that $\Sigma^{5}$ is a homotopy sphere, and therefore that $\Sigma^{5}=S^{5}$.

Since $D_{1}$ is an invariant neighborhood of the fixed point set and $D_{2}$ is a neighborhood of an orbit of type $Z_{m n}$, it is clear that the action on $S^{5}$ has all the properties listed in the first paragraph.

REMARKS. By taking the equivariant connected sum along the fixed point set, we get examples with any finite number of orbits of type $Z_{m n}$. The existence of actions of this type lends an additional difficulty to the problem of classifying smooth $S^{1}$ actions on $S^{5}$. For an action with a circle of fixed points and normal representation $t^{m}+t^{n}(m, n>1$ and relatively prime), it follows immediately from Smith theory and the obvious restrictions on the dimensions of the various fixed point sets that the only candidate for an additional orbit type is $\mathbf{Z}_{m n}$. The orbit space must be a homotopy four-sphere with the nonprincipal orbits corresponding to two embedded two-disks which have a common boundary and which may intersect transversely in their interiors. If we take a fixed orientation on $S^{5}$, then orientations can be assigned to the orbit space and the immersed two-sphere so that the selfintersection number will be an invariant of the equivariant concordance class of the action. It would be interesting to know if this integer, together with the pair $(m, n)$, determines the concordance class.

Similar actions exist in higher dimensions. Let $S_{0}$ denote our example on $S^{5}$ with a small, open five-disk about a fixed point removed. Take the product with the trivial action on $D^{k}$, and consider the boundary of $S_{0} \times D^{k}$. This will be an action on $S^{k+4}$, fixing $S^{k}$, and exhibiting an orbit structure similar to that of the action on $S^{5}$. By allowing nontrivial actions on the $D^{k}$ factor, and taking connected sums, more complicated examples can be realized. For instance, we can get an action on $S^{7}$ with a circle of fixed points, normal representation $t^{a}+t^{b}+t^{c}$ with $a, b$, and $c$ pairwise relatively prime, and having orbits of type $\mathbf{Z}_{a b}, \mathbf{Z}_{b c}$, and $\mathbf{Z}_{a c}$.

Finally, it has been pointed out by Reinhard Schultz that such examples can also be obtained by using Brieskorn varieties. Briefly, the construction is as follows. With $m$ and $n$ as above, let $S^{1}$ act on $S^{7}$ as the unit sphere in the representation $t^{m}+t^{n}+t^{m n}+1$. Let $V^{5}$ be the intersection of $S^{7}$ with the zeros of the complex polynomial $z_{0}^{n}+z_{1}^{m}+2 z_{2} z_{3}$. It can be verified that $V^{5}$ is a smooth invariant submanifold of $S^{7}$ which has the same fixed point set and orbit structure as our example. Now consider the following unitary change of coordinates:

$$
y_{2}=\left(z_{2}+z_{3}\right) / \sqrt{2}, \quad y_{3}=i\left(z_{2}-z_{3}\right) / \sqrt{2} .
$$

Through this change of coordinates, $V^{5}$ is identified with the variety in $S^{7}$ defined by the polynomial $z_{0}^{n}+z_{1}^{m}+y_{2}^{2}+y_{3}^{2}$. It then follows from $[2, \mathrm{p}$. 106] that $V^{5}$ is a five-sphere. This also shows that the circle action is embedded in the natural action of $S^{1} \times S O(2)$ on the Brieskorn sphere. (This method has been used by Bredon to construct exotic actions [1, p. 276], [3, p. 55].) 


\section{REFERENCES}

1. G. Bredon, Introduction to compact transformation groups, Academic Press, New York, 1972.

2. F. Hirzebruch and K. H. Mayer, $O(n)$-Mannigfaltigkeiten, Exotische Sphären und Singularitäten, Lecture Notes in Math., no. 57, Springer-Verlag, Berlin and New York, 1968.

3. P. S. Mostert (Editor), Proc. Conf. on Transformation Groups (New Orleans, 1967), Springer, New York, 1968, pp. 415-417. MR 41 \#2669.

Department of Mathematics, University of Chicago, Chicago, Iluinois 60637

Current address: Department of Mathematics, State University of New York at Stony Brook, Stony Brook, New York 11794 